\title{
A Documentation System to Save Time and Ensure Proper Application of the Fiberoptic Endoscopic Evaluation of Swallowing (FEES ${ }^{\circledR}$ )
}

\author{
Christiane Hey $^{\text {a Petra Pluschinskic }{ }^{c} \text { Soenke Stanschus }}{ }^{d}$ Harald A. Euler ${ }^{\mathrm{e}}$ \\ Robert A. Sader ${ }^{b}$ Susan Langmore ${ }^{f}$ Katrin Neumann ${ }^{a}$ \\ ${ }^{a}$ Clinic of Phoniatrics and Pediatric Audiology, and ${ }^{b}$ Clinic for Oral, Cranio-Maxillofacial and Facial Plastic \\ Surgery, University of Frankfurt am Main, Frankfurt am Main, 'Department of Geriatrics, Otto Fricke Hospital, \\ Bad Schwalbach, d Department of Dysphagia Treatment and Speech Therapy, SRH Hospital \\ Karlsbad-Langensteinbach, Karlsbad, e Institute of Psychology, University of Kassel, Kassel, Germany; \\ fBoston Med Center, Speech and Hearing Sciences, Boston University, Boston, Mass., USA
}

\section{Key Words}

Deglutition disorders · Fiberoptic endoscopic evaluation of swallowing $\cdot$ Documentation system $\cdot$ Editing time

\begin{abstract}
A properly performed fiberoptic endoscopic evaluation of swallowing $\left(\mathrm{FEES}^{\circledR}\right)$ is comprehensive and time-consuming. Editing times of FEES protocols and attempts for efficiency maximization are unknown. Here, the protocol editing times of completed FEES examinations were determined. The present study reports the time savings and quality gains of a newly developed documentation system tailored to the FEES standard of Langmore. Four independent examiners analyzed twelve videos of FEES procedures, six without and six with the documentation system. Effectiveness of the documentation system was evaluated according to the times for total evaluation, interpretation, documentation, report writing, and for report completeness. The documentation system reduced editing times and increased report completeness with large effect sizes. Averaged total evaluation time decreased from 42 to $27 \mathrm{~min}$, report completeness increased from 55 to $80 \%$. The use of the documentation system facilitates and improves the assessment of the swallowing process.

Copyright $\odot 2010$ S. Karger AG, Basel
\end{abstract}

\section{Introduction}

Transnasal flexible endoscopy has become a widely used and powerful tool to assess swallowing dysfunction in a variety of settings over an array of patients [1-3]. Performed as fiberoptic endoscopic evaluation of swallowing $\left(\mathrm{FEES}^{\circledR}\right)$ as defined by Langmore [4], endoscopy even is acknowledged as a gold standard for the diagnosis of oropharyngeal dysphagia and is often used as a benchmark for other methods [5]. Various studies demonstrated that FEES is as sensitive as or more sensitive than the videofluoroscopic study of swallowing (VFSS) with regard to the parameters penetration, aspiration, delay in swallowing initiation, and pharyngeal residue [6-8]. Hence, many European clinicians, oriented to the international literature [e.g. 9-11], have begun to use the FEES procedure in their daily routine.

With the increased use of FEES in daily practice, FEES, however, has turned out to be time-consuming and costly when performed and analyzed as required. Still, Aviv et al. [12] demonstrated that the FEES with sensory testing appeared to be more cost-effective than VFSS for the inpatient management of dysphagia. Although the FEES procedure itself is completed swiftly, the analysis is rather comprehensive, even for those with many years of clinical experience [13]. In turn, the editing time, that is, in-

\section{KARGER}

Fax +41613061234 E-Mail karger@karger.ch www.karger.com
Christiane Hey, MD

Clinic for Phoniatrics and Pediatric Audiology, University of Frankfurt am Main Theodor-Stern-Kai 7, House 7A

DE-60590 Frankfurt am Main (Germany)

Tel. +49696301 5775, Fax +49696301 5002, E-Mail Christiane.Hey@ kgu.de 
terpretation, documentation, and report writing has become time-consuming. Clinicians then have been left with the problem of trying to deal with the demands of health insurance providers, that is, more elaborate documentation while at the same time reducing costs $[14,15]$. The consequence of improving time efficiency in order to lower costs, however, has been often incomplete examination, documentation, and reports. Which parameters of FEES are dispensable without too much loss of evaluation quality? Langmore trademarked FEES in order to maintain quality and ensure a standard [16]. Although she established this standard, there is a lack of a consensus among clinicians on the FEES procedure [5]. Ideally, clinicians would have access to a tool that strictly adheres to Langmore's FEES protocol and is time-efficient. There is, however, a paucity of reports on editing times of FEES protocols and attempts for efficiency maximization.

In order to provide a time-efficient solution to document and report a FEES examination without compromising integrity, a documentation system was developed for this study. The aim of this study was to determine (1) time efficiency and (2) report completeness by the application of the proposed documentation system. For these purposes, the time needed for interpretation, documentation, and report writing were assessed. Additionally, time saved and improvement in report quality were quantified.

\section{Methods}

\section{Documentation System}

The documentation system presented here strictly adheres to the specifications of the FEES protocol [4]. For a structured and concise layout, the evaluation parameters are arranged in tabular form. Each part of the protocol is displayed in a task-tailored checklist of normal and pathological findings which also allows for free text entry. Table 1 depicts an exemplary part of the documentation form. Single issues, like anatomical structures or related functional tasks, are placed on the left side of the form. Figures are provided into which conspicuous findings may be drawn if it is too difficult to describe them precisely in the unified checklist. Normal findings are listed in the middle column. In order to highlight pathologies, the right column contains task-tailored checklists of common pathological findings as choice options.

The documentation system is divided into three parts in accordance with the FEES procedure, namely (1) anatomic-physiologic assessment, (2) direct examination of swallowing food and liquids, and (3) therapeutic maneuvers tried and their effects. The items of the anatomic-physiologic assessment and of the examination of swallowing are shown in appendices 1 and 2 . The item order corresponds to the FEES procedure.

The section on anatomic-physiologic assessment documents the essential morphological and functional prerequisites for the oropharyngeal swallowing process. With the provided checklists, alterations in surface mucosa and structural integrity of velum/ epipharynx, tongue base/mesopharynx, and larynx/hypopharynx can be described in detail. The checklists for the biomechanical functions include the parameters symmetry, range/amplitude, speed/briskness, and coordination. As a critical clinical parameter, the occurrence of oropharyngeal secretions, scored by the four-point rating scale of Murray et al. [17], are itemized separately with a specification of its amount, its color and viscosity, the patient's response, and its management. The spontaneous swallows are documented in number of swallows per minute. The last section of the anatomic-physiologic assessment consists of sensation and sensory testing and registers the patient's reaction to the presence of the endoscope or a light touch to the aryepiglottic folds on the right and left sides.

Part 2 of the FEES protocol is a direct examination of swallowing food and liquids and constitutes the core of the examination. It depicts the most central findings of a FEES examination which point to abnormal bolus passage patterns, that is, leaking, penetration/aspiration, and residues. These findings are evaluated with respect to different consistencies and bolus volume and described for the oral stage (from the endoscopic view) and the pharyngeal stage.

Penetration/aspiration is scored by the eight-point penetration-aspiration scale of Rosenbek et al. [18] which enables the comparison of FEES with VFSS because this rating scale is validated for both diagnostic procedures $[19,20]$.

The description of the preparatory and transit phase of the oral stage from the endoscopic view includes the lingual velar seal, the base of tongue movements during the preparatory stage and its retraction in the transport stage, and a final documentation of the entire duration of the oral stage.

Leaking during the oral preparatory stage is documented separately and specified for the bolus path, and is depicted in figures for the consistencies liquid, semisolid, and solid. Finally, possible penetration or aspiration events for each consistency are scored.

The pharyngeal stage, examined either as instructed swallow with measured bolus amount or as natural eating and drinking, is itemized (1) for the timeliness of the initiation of swallow, (2) for the location of the bolus at the onset of the swallow, (3) for penetration or aspiration, scored as above by the penetration-aspiration scale, with detailed indications about the time of occurrence (pre-, intra-, or postdeglutitive), and (4) for amount and location of residual material left after the swallow.

The third part about therapeutic maneuvers tried and their effects provides checklists for changes in bolus delivery and postures as well as different swallowing maneuvers (table 2).

\section{Evaluation Procedure}

Twelve digital video recordings of pathological swallowing processes were taken by the first author according to the FEES protocol with the ENT video endoscopy system EndoStrob-DX [Xion medical GmbH; video system: PAL/NTSC; sensor: CCD matrix with micro-lenses and mosaic filter, 1/3" $752(\mathrm{~h}) \times 582$ (v) pixels (PAL); output: S-Video, DVI-I, VGA IEEE/1394 (DV); input DVI-I, S-Video; illumination: min. 3 lux; filter OLPF; digital interfaces: IEEE/1394 FireWire DV: audio and video; light source: power $50 \mathrm{~W}$; color temperature $5.700 \mathrm{~K}$; camera head with integrated microphone, videoadapter: focal length $=22 \mathrm{~m}$; clip coupling] and a transnasal flexible endoscope $11101 \mathrm{RP} 2$, outside diameter: $3.5 \mathrm{~mm}$ (Karl Storz GmbH, Tuttlingen). 
Table 1. Exemplary part of the documentation form: morphological findings larynx and function

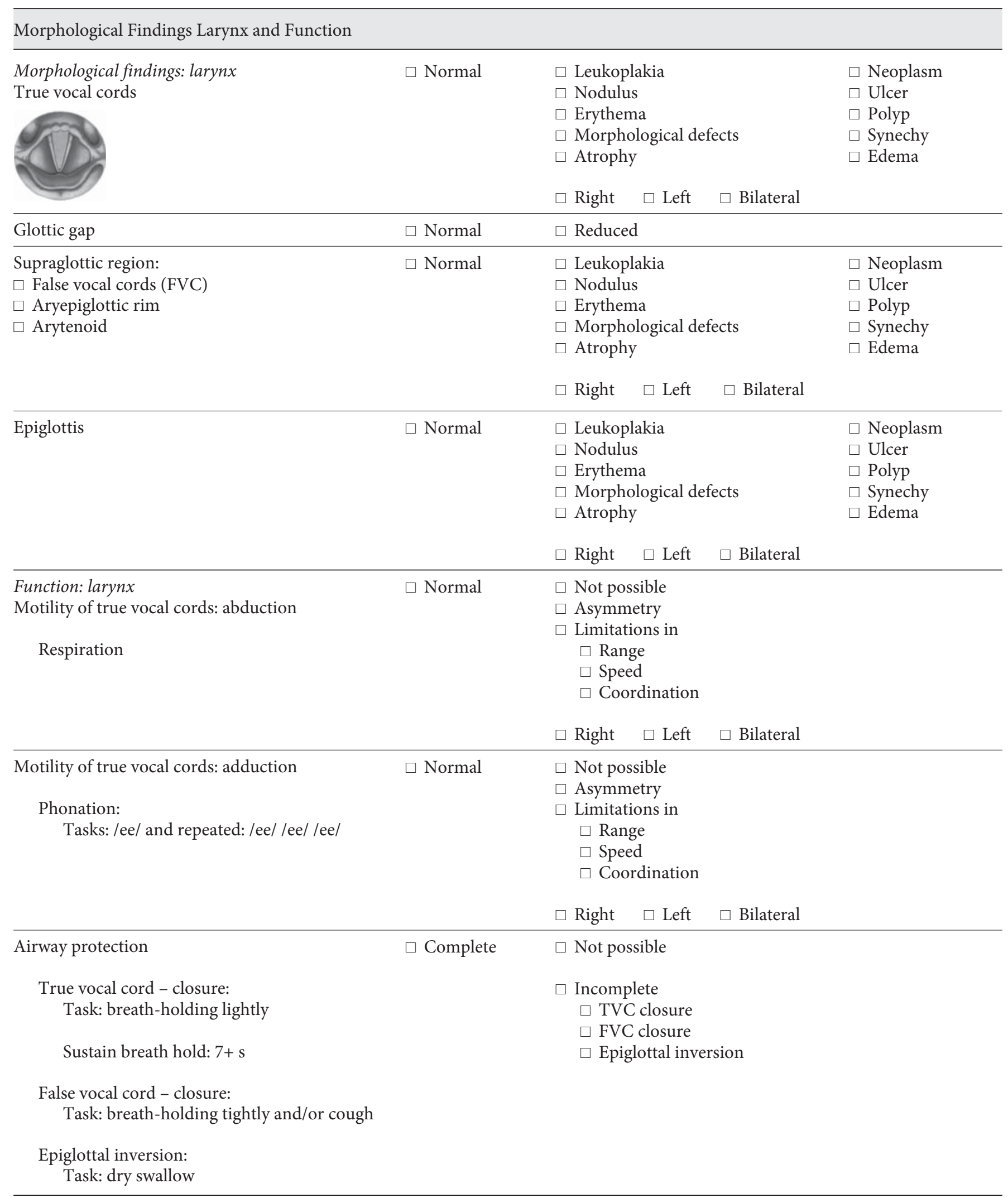


Table 2. Exemplary part of the documentation form: therapeutic maneuvers tried and their effect

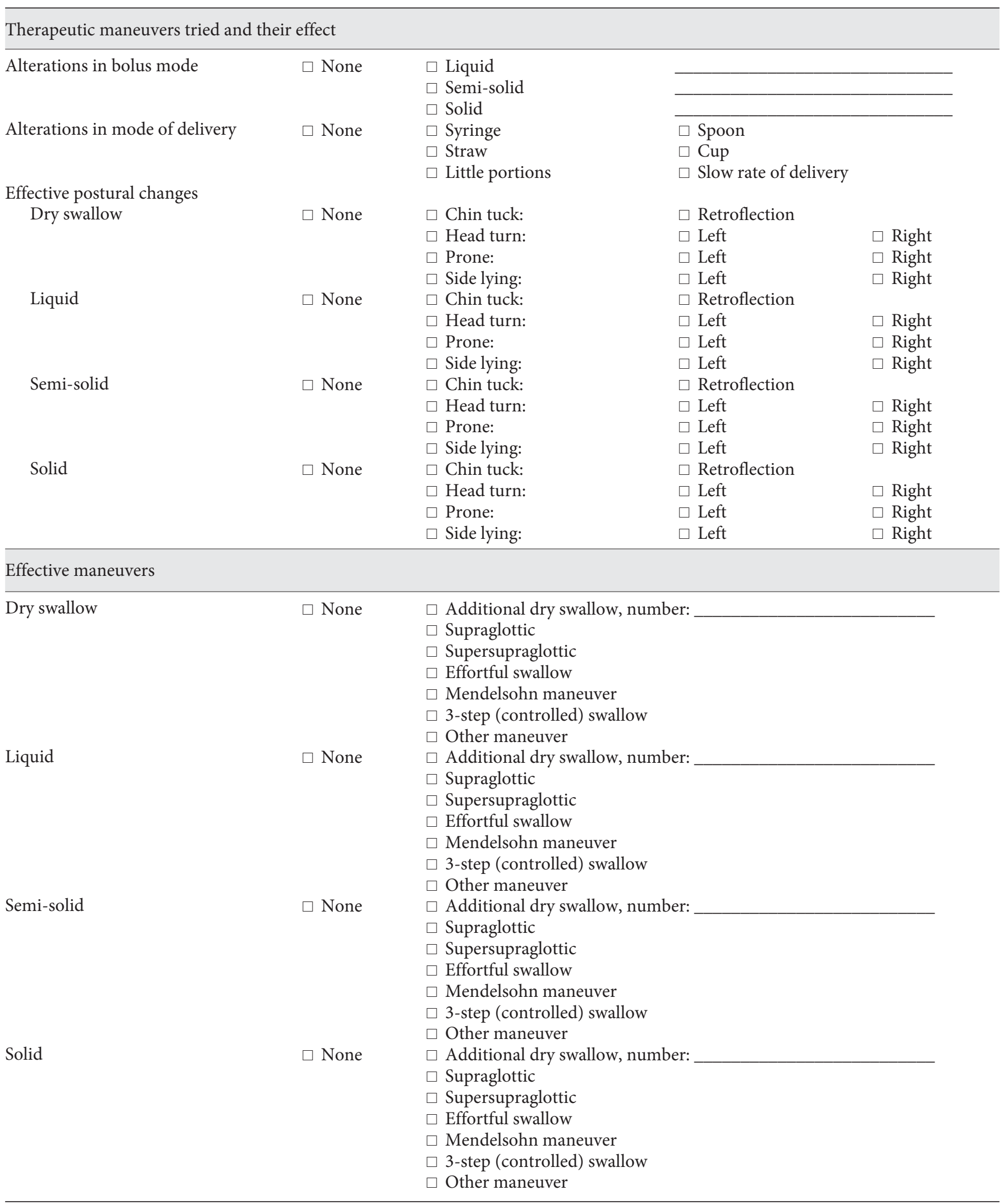


Table 3. Times for total evaluation, interpretation, documentation, and report writing as well as report completeness, for evaluation system $A$ and $B$ and examiner 1-4, and means and standard deviations over examiners

\begin{tabular}{|c|c|c|c|c|c|c|c|c|c|c|}
\hline \multirow[t]{3}{*}{ Parameter } & \multicolumn{10}{|c|}{ Evaluation system } \\
\hline & \multicolumn{2}{|c|}{ examiner 1} & \multicolumn{2}{|c|}{ examiner 2} & \multicolumn{2}{|c|}{ examiner 3} & \multicolumn{2}{|c|}{ examiner 4} & \multicolumn{2}{|c|}{ mean over examiners } \\
\hline & A & $\mathrm{B}$ & A & $\mathrm{B}$ & A & B & A & $\mathrm{B}$ & A & $\mathrm{B}$ \\
\hline Total evaluation time, min & 36.22 & 31.58 & 70.54 & 33.39 & 24.19 & 18.26 & 37.58 & 24.46 & $42.23 \pm 19.09$ & $27.12 \pm 8.14$ \\
\hline Time for interpretation, min & 10.56 & 16.31 & 24.06 & 23.36 & 7.08 & 6.34 & 10.00 & 9.12 & $13.02 \pm 9.32$ & $13.58 \pm 7.38$ \\
\hline Time for documentation, $\min$ & 9.21 & 7.17 & 22.09 & 6.14 & 7.41 & 5.16 & 11.49 & 5.35 & $12.45 \pm 6.36$ & $5.35 \pm 2.07$ \\
\hline Time for writing final report, $\min$ & 16.04 & 8.09 & 24.39 & 5.50 & 9.29 & 6.36 & 16.09 & 9.58 & $16.36 \pm 6.18$ & $7.38 \pm 3.21$ \\
\hline Report completeness, $\%$ & 48.4 & 82.1 & 70.3 & 90.2 & 39.0 & 69.4 & 62.2 & 77.0 & $54.98 \pm 14.65$ & $79.69 \pm 10.15$ \\
\hline
\end{tabular}

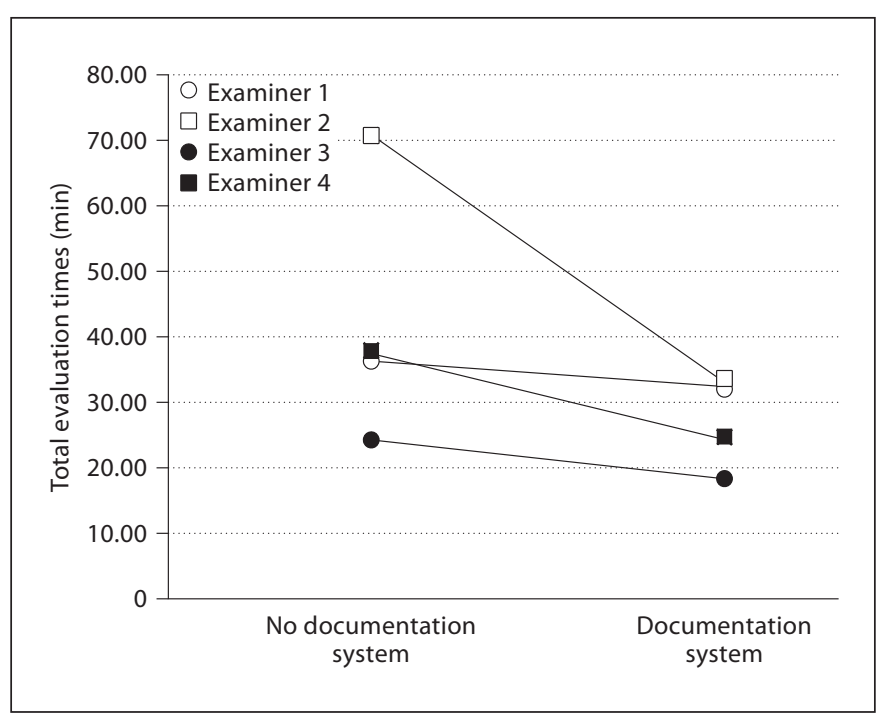

Fig. 1. Mean of the total evaluation times for the 4 examiners with and without documentation system.

The recordings were analyzed by 4 independent examiners, either with or without the documentation system. The examiners were two otolaryngologists, a speech and language pathologist and an oral and maxillofacial surgeon, each one from a different clinic and thus with different professional backgrounds. After being trained in the use of the documentation system, the examiners viewed the FEES video recordings. The depicted patients suffered from oropharyngeal dysphagia, six caused by head and neck cancer, six by neurological diseases (Parkinson's disease, stroke, cerebellar hemorrhage). Six recordings were assessed without the documentation system (evaluation system A) and six with the system (evaluation system B). The independent variables of the study were the evaluation system and the individual examiners. The films were arranged in a randomized balanced order to avoid serial effects. The mean film lengths differed slightly but not significantly between the two evaluation systems, as tested with the Mann-Whitney U test. For group A, the mean film length was
388 s (range 240-509 s), for group B 366 s (range 282-496 s). Depending on the individual need of the examiner, the films were viewed either in real time, with slowed speed, or frame-by-frame with or without repeated viewing.

Five criteria were the dependent variables: (1) total evaluation time (total time needed for interpretation, documentation, and writing a final report); (2) interpretation time; (3) documentation time; (4) time for writing a final report, and (5) completeness of reported parameters required by the original FEES protocol.

\section{Statistical Analysis}

A general linear model (GLM) two-factorial analysis was calculated to determine the effect of the evaluation system and examiner as well as the interaction effects on criteria 1-5. Although each examiner used both evaluation systems, they did so with a different film each time. Therefore, the independent variable of evaluation system (A or B) was considered a between-subject variable.

\section{Results}

Table 3 shows means and standard deviations of the different dependent variables, specified above as parameters 1-5, for each evaluation system and examiner.

As to total evaluation time, a 2 (Evaluation System A vs. B) by 4 (Examiners) GLM showed a main effect of Evaluation System $\left[\mathrm{F}(3,40)=57.01, \mathrm{p}<0.001, \eta^{2}\right.$ partial $=$ 0.59], a main effect of Examiners $[\mathrm{F}(3,40)=41.07, \mathrm{p}<$ $\left.0.001, \eta_{\text {partial }}^{2}=0.76\right]$, and a significant interaction Evaluation System $\times$ Examiners $[\mathrm{F}(3,40)=14.30, \mathrm{p}<0.001$, $\eta_{\text {partial }}^{2}=0.52$; fig. 1 ).

In order to determine the contributions of the single time components (time for interpretation, documentation, report writing) to the total evaluation time, a GLM was calculated for each of them. For the interpretation time, neither the main effect of the Evaluation System nor the interaction Evaluation System $\times$ Examiners was sig- 


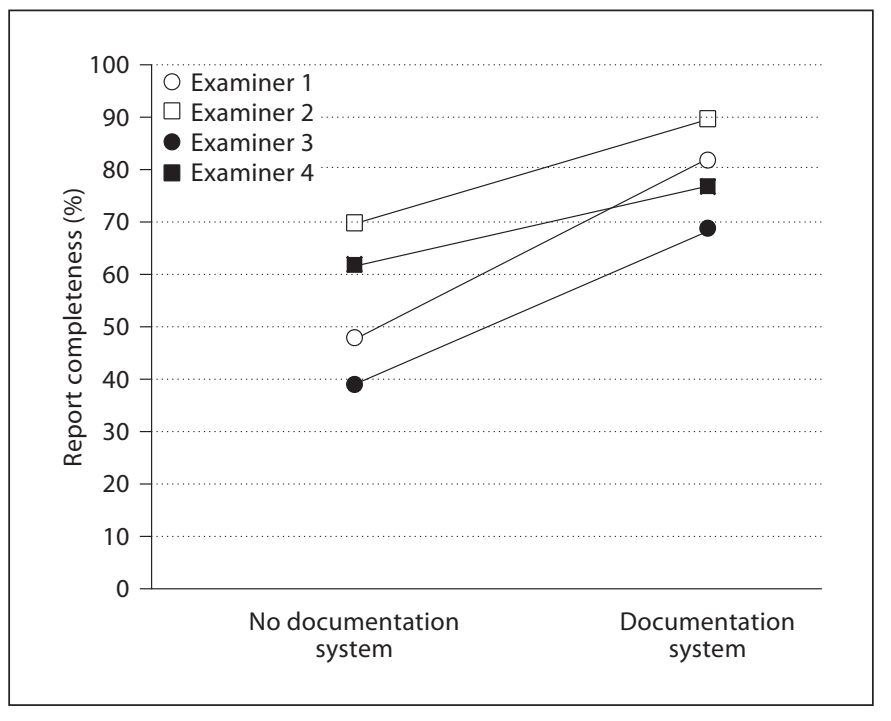

Fig. 2. Mean of the report completeness for the 4 examiners with and without documentation system.

nificant. The main effect of Examiners, however, was significant $\left[\mathrm{F}(3,40)=19.78, \mathrm{p}<0.001, \eta_{\text {partial }}^{2}=0.60\right]$. For documentation time, all three effects were significant: Evaluation System $\left[\mathrm{F}(3,40)=76.62, \mathrm{p}<0.001, \eta_{\text {partial }}^{2}=\right.$ $0.66]$, Examiners $\left[\mathrm{F}(3,40)=12.15, \mathrm{p}<0.001, \eta_{\text {partial }}^{2}=\right.$ $0.48]$, and Evaluation System $\times$ Examiners $[\mathrm{F}(3,40)=$ 20.57, $\left.\mathrm{p}<0.001, \eta_{\text {partial }}^{2}=0.61\right]$. Also for report time, all three effects were significant: Evaluation System $\left[\mathrm{F}(3,40)=92.57, \mathrm{p}<0.001, \eta_{\text {partial }}^{2}=0.70\right]$, Examiners $\left[\mathrm{F}(3,40)=10.47, \mathrm{p}<0.001, \eta_{\text {partial }}^{2}=0.440\right]$ and the interaction Evaluation System $\times$ Examiners $[\mathrm{F}(3,40)=13.76$, $\left.\mathrm{p}<0.001, \eta_{\text {partial }}^{2}=0.51\right]$.

For the report completeness, the main effect of Evaluation system was significant $[\mathrm{F}(3,40)=121.33, \mathrm{p}<0.001$, $\left.\eta_{\text {partial }}^{2}=0.75\right]$, as was the main effect of Examiners $\left[\mathrm{F}(3,40)=23.11, \mathrm{p}<0.001, \eta_{\text {partial }}^{2}=0.63\right]$. The interaction effect Evaluation System $\times$ Examiners $[\mathrm{F}(3,40)=$ 3.89, $\mathrm{p}<0.02, \eta_{\text {partial }}^{2}=0.23$ ] was significant (fig. 2).

\section{Discussion}

The employment of the presented documentation system in FEES reduced the editing time considerably and enhanced the completeness of the evaluation. In addition, the documentation system decreased the large betweenexaminer differences of both parameters. Thus, a tool has been developed which facilitates, unifies, and improves the assessment of a swallowing process, irrespective of examiner background.

When no documentation system was used, total evaluation time ranged from 24 (examiner 3) to $70 \mathrm{~min}$ (examiner 2), and report completeness from 39 (examiner 3 ) to $70 \%$ (examiner 2). The mean total evaluation time across the examiners decreased from 42 to $27 \mathrm{~min}$ by the use of the documentation system. A partial $\eta^{2}$ larger than 0.14 is considered a large effect size [21]; in this study, a partial $\eta^{2}$ of 0.58 for the total evaluation time was gained by the documentation system. This reduction in total time was mainly due to time savings for the documentation and report writing. The mean interpretation time, however, increased slightly from 13 to $14 \mathrm{~min}$ by the use of the documentation system. The reduction in the documentation time was due to the provided display format of the evaluation scheme. The uniform display format facilitates the preparation of the final report.

The time needed for total evaluation, interpretation, and documentation, as well as report completeness varied with individual examiner. A significant interaction between employed documentation system and examiner was found for time needed for total evaluation, documentation, and report writing as well as report completeness. Despite this interaction, an advantage for using the new documentation system was seen for all 4 examiners, even though not equally across all parameters. All 4 examiners profited with respect to report completeness. One of them (examiner 2), who already wrote thorough reports, was able to shorten her time by more than $50 \%$ using the documentation system.

This study has several limitations. The number of examiners was too small to establish time norms. However, study participants were difficult to locate because in Germany as well as in many other European countries dysphagia is a young research field, and only few clinicians are specialized in swallowing disorders. Furthermore, the aim of the study was not to investigate agreement between and within examiners. Instead, our primary aim was to reduce editing time by maintaining report completeness according to FEES standard. If report completeness varies between examiners, as it does especially in the absence of a standard documentation system, the agreement between examiners can only be calculated for those report parameters shared by examiners but not for those parameters reported by one examiner but ignored by the other. It would be valuable to use the current standard documentation system to determine inter-judge reliability in future studies. 


\section{Conclusion}

Without a standard documentation system, the time and quality of written reports from FEES examinations were inefficient and incomplete. Using the presented documentation system developed in this study, both time effectiveness and the quality of the analysis of the FEES improved significantly. The mean total evaluation time across all 4 examiners decreased from 42 to $27 \mathrm{~min}$, and the completeness of the final report improved from 55 to $80 \%$.

The documentation system for FEES examinations is offered as a tool to standardize the reporting of the exam as well as to reduce time needed to write it and improve the quality and thoroughness of the report. Standardization is a necessary step in validating a procedure, and our documentation system is a contribution toward that goal.

\section{Appendix 1}

Part 1 of FEES: Evaluated Parameters of Anatomic-Physiologic Assessment

Evaluation of morphological findings and function:

Velum and epipharynx, base of tongue and mesopharynx, larynx and hypopharynx

Checklist entries for morphological findings:

Neoplasm, ulcer, erythema, morphological defects,

hypertrophy, atrophy, asymmetry involuntary movements at rest

Checklist entries for function (movement qualities for every evaluated task):

Symmetry, limitations in: speed, range and coordination

Evaluated function and tasks:

Velar and lateral wall movements

Tasks: velopharyngeal closure by dry swallow and phonation

Retraction of base of tongue

Tasks: dry swallow, repeating of postvocalic /l/ word like 'all'

Movement of pharynx constrictors and longitudinal muscles Tasks: phonation of strained loud high /ee/ and repeated lee/
Abduction and adduction of true vocal cords Tasks: respiration and phonation: sustained and repeated lee/

Airway protection

Tasks: true vocal folds closure: breath-holding lightly (also, hold for $7+s$ ), false vocal folds closure: breath-holding tightly, cough, epiglottal inversion: dry swallow

Secretions and spontaneous swallowing:

Locations of oropharyngeal secretions, appearance of

secretions (color, viscosity), awareness to secretions, patient's response, frequency of spontaneous swallows

Sensation and sensory testing.

Response to presence of endoscope, response to light touch to junction of aryepiglottic folds and arytenoid cartilages

\section{Appendix 2}

Part 2 of FEES: Direct Examination of Swallowing Food and Liquids

Oral stage (fiberoptic endoscopic view):

Competence of linguovelar seal, base-of-tongue movements, base-of-tongue retraction, bolus transport into the pharynx, duration of complete oral stage

Spillage of bolus during oral preparatory stage (for the different bolus consistencies: liquid, semi-solid, solid): bolus path, penetration and/or aspiration

Pharyngeal stage:

Checklist entries for different bolus consistencies and bolus volumes:

Measured bolus volumes with instructions to swallow: Bolus path, swallow initiation, penetration/aspiration, pre-, intra-, and postdeglutitive

Natural drinking and eating behavior: Bolus path, swallow initiation, penetration/aspiration, pre-, intra-, and postdeglutitive

Hyolaryngeal elevation: epiglottal inversion (impression from all swallows)

Residues and management:

Checklist entries include the different bolus consistencies and bolus volumes:

Location of residues, amount of residues, awareness to residues, clearing forces, effectiveness of clearing force

\section{References}

1 Warnecke T, Ritter MA, Kroger B, Oelenberg S, Teismann I, Heuschmann PU, Ringelstein EB, Nabavi DG, Dziewas R: Fiberotic endoscopic dysphagia severity scale predicts outcome after acute stroke. Cerebrovasc Dis 2009;28:283-289.

-2 Hafner G, Neuhuber A, Hirtenfelder S, Schmedler B, Eckel HE: Fiberoptic endoscopic evaluation of swallowing in intensive care unit patients. Eur Arch Otorhinolaryngol 2008;265:441-446.

- 3 Dworkin JP, Hill SL, Stachler RJ, Meleca RJ, Kewson D: Swallowing function outcomes following nonsurgical therapy for advancedstage laryngeal carcinoma. Dysphagia 2006; 21:66-74.

4 Langmore SE: Scoring a FEES examination; in Langmore SE (ed): Endoscopic Evaluation and Treatment of Swallowing Disorders. New York, Thieme, 2001, pp 101-143.

5 Hiss SG, Postma GN: Fiberoptic endoscopic evaluation of swallowing. Laryngoscope 2003;113:1386-1393.

6 Langmore SE, Schatz K, Olson N: Endoscopic and videofluoroscopic evaluations of swallowing and aspiration. Ann Otol Rhinol Laryngol 1991;100:678-681. 
7 Wu CH, Hsiao TY, Chen JC, Chang YC, Lee SY: Evaluation of swallowing safety with fiberoptic endoscope: comparison with videofluoroscopic technique. Laryngoscope 1997; 107:396-401.

8 Leder SB, Sasaki CT, Burrell MI: Fiberoptic endoscopic evaluation of dysphagia to identify silent aspiration. Dysphagia 1998;13:1921.

-9 Langmore SE, Schatz K, Olsen N: Fiberoptic endoscopic examination of swallowing safety: a new procedure. Dysphagia 1988;2:216219.

-10 Aviv JE, Kim T, Thomson JE, Sunshine S, Kaplan S, Close LG: Fiberoptic endoscopic evaluation of swallowing with sensory testing (FEESST) in healthy controls. Dysphagia 1998;13:87-92.

$\checkmark 11$ Leder SB, Espinosa JF: Aspiration risk after acute stroke: comparison of clinical examination and fiberoptic endoscopic evaluation of swallowing. Dysphagia 2002;17:214-218.
12 Aviv JE, Sataloff RT, Cohen M, Spitzer J, Ma G, Bhayani R, Close LG: Cost-effectiveness of two types of dysphagia care in head and neck cancer: a preliminary report. Ear Nose Throat J 2001;80:553-558.

13 Warnecke T, Teismann I, Oelenberg S, Hamacher C, Ringelstein EB, Schäbitz WR, Dziewas R: Towards a basic endoscopic evaluation of swallowing in acute stroke - identification of salient findings by the inexperienced examiner. BMC Med Educ 2009;9:13.

14 Noack M, Schneider T, Nosper M: Eva-Reha: a computer software supporting outcomebased quality management in medical rehabilitation. Gesundheitswesen 2005;67:289295.

15 Bassler M, Nosper M, Follert P, Böwering L, Polak U: Data sources for continual quality improvement in medical rehabilitation - the QS-Reha procedure of the statutory health insurance funds and the Eva-Reha documentation system of MDK Rhineland-palatinate. Rehabilitation 2007;46:155-163.

16 Langmore SE: Evaluation of oropharyngeal dysphagia: which diagnostic tool is superior? Curr Opin Otolaryngol Head Neck Surg 2003;11:485-489.
17 Murray J, Langmore SE, Ginsberg S, Dostie $\mathrm{Al}$ : The significance of accumulated oropharyngeal secretions and swallowing frequency in predicting aspiration. Dysphagia 1996; 11:99-103.

18 Rosenbek JC, Robbins JA, Roecker EB, Coyle JL, Wood JL: A penetration-aspiration scale. Dysphagia 1996;11:93-98.

19 Colodny N: Interjudge and intrajudge reliabilities in fiberoptic endoscopic evaluation of swallowing (FEES) using the penetrationaspiration scale: a replication study. Dysphagia 2002;17:308-315.

20 Kelly AM, Drinnan MJ, Leslie P: Assessing penetration and aspiration: how do videofluoroscopy and fiberoptic endoscopic evaluation of swallowing compare? Laryngoscope 2007;117:1723-1727.

21 Bortz J, Döring N: Forschungsmethoden und Evaluation. Berlin, Springer, 2002. 